\title{
Thérapie EMDR et psycho-oncologie : un pont entre le corps et l'esprit
}

\author{
Elisa Faretta \\ SIMP Association italienne pour la médecine psychosomatique, Milan, Italie \\ Association EMDR Italie \\ Cristina Civilotti \\ Département de psychologie, université de Turin, Italie \\ Centre de thérapie EMDR spécialisé dans l'anorexie et les troubles des conduites alimentaires, Milan, Italie
}

\begin{abstract}
Parmi les maladies potentiellement mortelles, le cancer est l'une des plus traumatisantes et des plus génératrices de détresse. II impacte le sens même de l'identité de l'individu et perturbe chez lui des caractéristiques essentielles, en rapport direct avec la conscience de soi et le caractère unique de la personne. II attaque l'intégrité physique des patients, amenant la question de la mort au premier plan, et peut mettre en cause directement leur sentiment d'appartenance aux micro- et macro-systèmes sociaux autour d'eux. Cet article souligne l'importance de comprendre que souffrance psychologique et douleur physique sont étroitement interconnectées et, dans le contexte de la psycho-oncologie, propose une perspective clinique basée sur l'approche de désensibilisation et de retraitement par les mouvements oculaires (EMDR), approche pour laquelle l'événement qu'est le cancer est inscrit dans l'histoire de vie du patient. L'EMDR est une approche thérapeutique fondée sur le modèle du traitement adaptatif de l'information (TAI). Le modèle TAI postule que la psychopathologie apparait quand des expériences non traitées sont enregistrées dans leur propre réseau neuronal et se trouvent dans l'impossibilité de se connecter à d'autres réseaux plus adaptatifs. Dans cette perspective, on suppose que le noyau de la souffrance clinique est enchâssé dans ces souvenirs qui demeurent en suspens de façon dysfonctionnelle. Selon des articles scientifiques récents que nous présentons dans cet article, il semble que les traumas du passé et les traumas liés au cancer entretiennent un cercle vicieux entre la santé psychologique et physique de l'individu, et l'objectif de la thérapie EMDR est de briser ce cercle. De récentes recherches scientifiques avancent l'hypothèse que le thérapie EMDR est efficace au niveau psychologique comme au niveau physique. Cependant, à cause de l'hétérogénéité générale des protocoles de recherche, les résultats rapportés dans cet article soulignent la nécessité de mener d'autres recherches contrôlées afin de permettre une analyse plus complète.
\end{abstract}

Mots-clés : désensibilisation et retraitement par les mouvements oculaires (EMDR); psycho-oncologie ; cancer ; traitement adaptatif de l'information (TAI) ; psychothérapie.

$\mathbf{U}$ n diagnostic de cancer peut provoquer un bouleversement spectaculaire dans la vie d'une personne et affecter ses émotions, son raisonnement, sa santé physique, ses relations ainsi que la dimension spirituelle de son existence (Grassi, Biondi \& Costantini, 2009). La représentation cultu- relle du cancer comprend souvent la vision de grandes souffrances physiques, voire de la mort, c'est-à-dire des faits habituellement perçus comme des possibilités lointaines ou des tragédies qui concernent les autres. De plus, la maladie change totalement la nature des relations entre les patients atteints d'un cancer, leurs

This article originally appeared as Faretta, E. \& Civilotti, C. (2016). EMDR Therapy in psycho-oncology: A Bridge Between Mind and Body. Journal of EMDR Practice and Research, 10(3), 138-152. Translated by François Mousnier-Lompré. 
familles et l'environnement social plus large, perturbant les règles habituelles de réciprocité et de soutien mutuel (Bury, 1982 ; Townsend, Wyke \& Hunt, 2006).

Holland (2004), qui est largement reconnu comme l'un des fondateurs de la psycho-oncologie, souligne que pendant des siècles, un diagnostic de cancer représentait une honte parmi le public, les patients et leurs familles, et au sein même du corps médical qui ne communiquait que rarement le diagnostic de la maladie à ceux qui en étaient atteints. La pratique consistant à " garder le secret " envers les patients empêchait à la fois de parler avec eux de leurs émotions ou des problèmes psychologiques liés à la maladie et d'adopter des stratégies appropriées leur permettant de faire face à la maladie et à la menace de mort qu'elle comporte. Ce n'est qu'au cours des dernières décennies que le droit des patients à accéder aux informations et la diminution de la stigmatisation accompagnant le diagnostic du cancer ont permis un dialogue plus ouvert et une plus grande reconnaissance de l'impact émotionnel de la maladie sur l'état mental du patient.

Bien que la littérature scientifique (Bultz \& Holland, 2006) et les directives internationales (National Comprehensive Cancer Network, 2013) se concentrent de plus en plus sur l'importance de reconnaître et de gérer la souffrance psychologique liée au cancer, le fardeau que représente l'épreuve du cancer pour les patients demeure souvent non reconnu et rarement diagnostiqué (Fallowfield, Ratcliffe, Jenkins \& Saul, 2001 ; Holland, 2004 ; Söllner et coll., 2001). Les patients montrant des symptômes ouvertement liés à la souffrance mentale ne reçoivent pas toujours le traitement adéquat (Morasso \& Tomamichel, 2005) et, se sentant confus, impuissants et coupables, adoptent une tendance au retrait émotionnel. En outre, le personnel médical se focalise parfois trop sur les aspects médicaux, au détriment d'une relation axée davantage sur l'expérience émotionnelle des patients, ce qui laisse de nombreuses émotions non dites. Avec le temps, ces émotions resteront non verbalisées et risquent de "se cristalliser " en souvenirs traumatiques (Holland, 1989 ; Mager \& Andrykowsky, 2002).

Les patients atteints du cancer, contre leur volonté, peuvent se voir devenir des individus passifs assistés par des proches qui commencent à prendre des décisions à leur place une fois que le diagnostic a été prononcé. Il est nécessaire d'adopter une approche en plusieurs phases, impliquant d'une part le traitement des symptômes physiques et, d'autre part, une assistance psychologique axée sur les aspects émotionnels liés à l'angoisse, au rejet et à la tristesse. Ces émotions requièrent des interventions spécifiques visant à renforcer les fonctions physiques restées intactes et la résilience psychologique du patient au cours des divers stades de la maladie. Notre article vise à établir l'existence d'un lien entre les aspects psychologiques et physiques de l'expérience humaine, et la façon dont l'approche de désensibilisation et de retraitement par les mouvements oculaires (EMDR), dans des contextes médicaux, peut être efficace auprès des patients atteints du cancer.

\section{Les implications psychosociales du cancer}

\section{L'impact sur l'individu}

D'un point de vue clinique, le cancer a un impact traumatique énorme sur la vie d'un individu. En tant que maladie chronique et mortelle, qui s'accompagne d'une symptomatologie souvent douloureuse, de fréquentes périodes d'hospitalisation et de procédures thérapeutiques invasives, le cancer ne représente pas uniquement une menace pour la vie du patient, mais également pour l'ensemble de son système psychologique (Borio \& Torta, 2007 ; Drageset, Lindstrom, Giske \& Underlid, 2011 ; Granieri et coll., 2013 ; O’Connor, Wicker \& Germino, 1990). Il affecte l'intégrité psychophysique, la qualité de vie et les chances de survie de la personne (Holzner, Giesinger \& Efficace, 2014) de même que son bien-être subjectif (Hou \& Lam, 2014 ; Tessier, Lelorain \& Bonnaud-Antignac, 2012). Le cancer génère également chez le malade un sentiment de vulnérabilité, une perte de contrôle et d'autonomie personnelle ainsi qu'un sentiment de désespoir (Kenne Sarenmalm, Browall, Persson, FallDickson \& Gaston-Johansson, 2013 ; Rodin et coll., 2009), en plus d'une symptomatologie purement post-traumatique : pensées intrusives, phénomène d'évitement et augmentation de l'hyperactivation (Baider \& Kaplan De-Nour, 1997 ; Brewin, Watson, McCarty, Hyman \& Dayson, 1998 ; Civilotti et coll. 2015 ; Kangas, Henry \& Bryant, 2002 ; Matsuoka, Nagamine \& Uchitomi, 2006 ; Smith, Redd, Peyser \& Vogl, 1999). Les aspects psychologiques liés à la maladie, y compris son impact social, risquent d'être partiellement négligés si l'effort principal n'est consacré qu'à « soigner » le cancer, comme si le patient était un appareil tombé en panne.

Le cancer impacte le sens même de l'identité de l'individu et perturbe chez lui des caractéristiques essentielles, en rapport direct avec la conscience de soi et le caractère unique de la personne. Il attaque son intégrité physique et fait passer la mort au premier plan ; ses effets et son traitement entraînent des modifications spectaculaires de l'image corporelle du patient, y compris des transformations de la façon 
dont il perçoit les parties de son propre corps. Chaque étape, depuis le diagnostic jusqu' au traitement et à la rémission, met en jeu des mécanismes de défense et des réponses émotionnelles qui influencent la façon dont le patient produit du sens par rapport à sa maladie (Grassi et coll., 2009).

Le modèle de Kübler-Ross (1969) décrit parfaitement les différentes étapes conduisant un patient à l'acceptation de la maladie. Le déni et l'incrédulité sont les premières réactions. Plus tard, la réalité ouvre la voie à des réactions de colère et souvent de protestation furieuse, suivies de sentiments plus dépressifs qui permettent finalement au sujet de reconnaître et d'accepter la maladie. Une maladie mortelle peut ainsi amener des questions existentielles jusque-là négligées à être abordées d'un point de vue religieux ou spirituel. Le cancer peut aussi menacer directement le sentiment d'appartenance du patient au microsystème que composent sa famille, ses amis proches et ses voisins. Par la suite, les sentiments d'aliénation peuvent s'étendre au macrosystème plus large de l'environnement social, du travail et de la participation à divers groupes sociaux (Grassi et coll., 2009).

\section{L'impact sur la famille}

En retour, les proches doivent réajuster leurs comportements et leurs relations au sein de la famille, de même qu'au sein de leur entourage. Ils ont à faire face à de nouvelles responsabilités, doivent jouer des rôles inattendus, déterminés par la maladie de l'être cher, dont la plus grande dépendance à leur endroit les oblige à partager sa peur de la mort et d'être témoins de tous ses changements physiques. Quelle que soit la personne principalement en charge de la personne malade, la perte d'autonomie du patient, ses douleurs, son épuisement et les effets secondaires du traitement sont un poids important pour tous les membres de la famille.

Les familles doivent faire face à divers niveaux de difficulté. Il faut redéfinir, repenser, revoir le sens qu'on accordait à la vie et à sa propre existence. Les capacités d'attention et d'adaptation des familles sont déplacées de force vers la maladie et sont ébranlées par des sentiments de perte, d'abandon, d'angoisse de séparation, d'anxiété, d'inévitabilité et de culpabilité. Les rôles familiaux doivent inévitablement changer et, à mesure que le temps passe, les individus devront faire face à davantage de pression physique : fatigue, épuisement et difficulté à s'endormir ou à se réveiller. Très souvent, les familles sont assaillies par des barrières non médicales, telles que des difficultés concernant les informations, les finances, le travail et le foyer. Les interactions avec les autres, particulièrement avec les institutions, peuvent devenir problématiques ; des difficultés de communication avec les équipes de santé peuvent se produire, avec des ambiguïtés, des conflits et des malentendus, de même que des problèmes d'organisation et des manques dans l'apport des traitements.

\section{Influence des facteurs de stress de la petite enfance et des facteurs biopsychosociaux sur le fonctionnement immunitaire et la santé}

En 1986, l’Organisation mondiale de la santé, de même que la plupart des systèmes de santé officiels du monde, ont incorporé le modèle biopsychosocial (Engel, 1977) à leur cadre directeur, se focalisant sur des perspectives multifactorielles qui évaluent l'interaction entre l'organisme individuel, l'environnement et les systèmes sociaux. Plus tard, les connexions entre les aspects psychologiques et physiologiques des risques et de la progression du cancer furent étudiées via l'approche psycho-neuro-immunologique, selon laquelle une activation permanente de l'axe hypothalamique-hypophyso-surrénalien (axe HHS) dans la réponse au stress chronique est susceptible d'affaiblir la réponse immunitaire et d'influencer l'installation et l'expansion de certains types de cancer (Reiche, Nunes \& Morimoto, 2004).

Cette perspective soutient le modèle de traitement adaptatif de l'information (TAI) (Shapiro, 2001), basé sur les principes théoriques et structurels de l'EMDR. Le modèle TAI postule qu'il existe un système physiologique inné, programmé pour transformer l'information perturbatrice en résolution adaptative et en intégration psychologique saine. Un trauma peut perturber le système de traitement de l'information, enregistrant dans la mémoire sa perception originelle, comme on le voit dans l'état de stress post-traumatique (ESPT ; Shapiro, 1995). En tant que méthode thérapeutique, l'EMDR reconnait l'importance des émotions et des réactions émotionnelles comme parties intégrantes d'un processus qui guide et module les interprétations cognitives et les élaborations de sens de l'individu.

Nous avons fait l'hypothèse que l'élaboration et l'intégration des émotions et des sensations physiques liées à des souvenirs traumatiques, si elles sont retraitées avec l'EMDR, pourraient être stimulées assez efficacement pour permettre d'en remodeler et reconsolider les éléments les plus perturbants, qu'ils soient 
liés à la maladie ou à des expériences douloureuses du passé. En fonction du stade atteint par la maladie et de l'historique traumatique personnel qui lui est associé, différentes cibles EMDR peuvent être choisies pour traiter ces patients, ainsi que le décrit le protocole EMDR spécifique aux patients atteints du cancer (Faretta, 2014).

Pour le décrire brièvement, le protocole EMDR en huit phases traite les souvenirs dysfonctionnels que le modèle TAI suppose " figés » dans des réseaux dysfonctionnels du cerveau. En psycho-oncologie, le traitement EMDR se focalise sur tous les souvenirs dysfonctionnels liés au cancer, depuis le diagnostic jusqu'au moment présent, et sur les trois perspectives de la vie du patient : passé, présent et futur. Le traitement se concentre sur l'élaboration de la résilience, le renforcement de la régulation émotionnelle et des capacités d'adaptation du patient (pour une discussion plus détaillée sur le protocole clinique EMDR sur le cancer, voir Faretta, 2014, 2015 ; Faretta, Agazzi, Poli, Sacchezin \& Zambon, 2013 ; Faretta \& Sacchezin, 2015).

\section{Les influences des expériences négatives de l'enfance sur le fonctionnement psychologique et physique}

L'article scientifique de Felitti et coll. de 1998 peut être considéré comme une étude révolutionnaire de santé publique. Pour la première fois, cette étude affirmait qu'il existe une relation claire entre les expériences négatives de l'enfance (ENE) et l'apparition de maladies et de comportements à risque à l'âge adulte. Les ENE sont décrites comme le résultat de dysfonctionnements familiaux capables de produire des traumas chez l'enfant, à la fois directement (p. ex. agression psychologique, physique ou sexuelle) et indirectement (p. ex. négligences graves, exposition à la toxicomanie, à la maladie mentale, à la violence conjugale ou à des comportements criminels d'un parent ; Felitti \& Anda, 2010 ; Liotti \& Farina, 2012 ; Lyons-Ruth, Bronfman \& Parson, 1999). La parution de ce rapport a changé définitivement l'approche de la santé et de la psychothérapie ; il était désormais impossible d'ignorer le lien entre les événements traumatiques et leur impact sur la santé.

\section{Expériences négatives de l'enfance et développement neurobiologique de l'enfance}

Les ENE ont une influence très importante sur le développement neurobiologique de l'enfant et peuvent entraîner des séquelles à vie (Rees, 2007). Le trauma peut causer des changements neuronaux et hormonaux durables, qui modifient la structure et le fonctionnement du cerveau (Anda et coll., 2006 ; Benedetti et coll., 2011) ; Boyce \& Ellis, 2005 ; Carrion \& Wong, 2012 ; Danese \& McEwen, 2012 ; Matz et coll., 2010 ; Meaney, 2010 ; Oberlander et coll., 2008 ; Reiche et coll., 2004 ; Roth \& Sweatt, 2011 ; Schonkoff et coll., 2012 ; Van der Kolk, 2003); ceux-ci peuvent à leur tour affecter les émotions, les comportements et la cognition. Le manque de ressources internes et externes pour un TAI peut accroître la probabilité d'un traumatisme (Cotraccia, 2012). Ce point est étroitement lié à la théorie de l'attachement.

Le système d'attachement, comme le dit Bowlby (1988), est actif «du berceau à la tombe » et est activé chaque fois qu'une situation difficile et douloureuse se produit, dans laquelle le sujet se sent vulnérable. Schore $(2003,2009)$ décrit les interactions relationnelles précoces avec la personne en charge de l'enfant comme une situation susceptible de produire une désorganisation de l'attachement. Dans cette configuration, une attitude parentale ouvertement hostile ou un parent impuissant peuvent entraîner chez l'enfant la peur et l'impuissance, état déterminé par le paradoxe insoluble où la personne en charge de l'enfant est simultanément une source de danger et un protecteur (Lambruschi, Lenzi \& Leoni, 2004 ; Liotti \& Farina, 2011). Au sein de cette configuration, comme dans la réponse au trauma, il y a une activation simultanée, mais incompatible des mécanismes de défense et du système d'attachement (Liotti \& Farina, 2011). Ainsi que l'a expliqué Schore (2000), la relation avec la mère régule non seulement les affects de l'enfant, mais également la production hormonale et neurohormonale, qui influencent les systèmes d'activation, encodés génétiquement, qui sont impliqués dans le développement structurel de certaines régions cérébrales essentielles pour le développement social et émotionnel de l'enfant (en particulier le cortex orbital préfrontal dans l'hémisphère droit et le système limbique).

L'hémisphère droit est principalement impliqué dans l'encodage des sensations corporelles et des souvenirs émotionnels dès la naissance, connectant le circuit limbique émotionnel au néocortex via le cortex orbitofrontal. Une exposition précoce et prolongée à un stress causé par un trauma et/ou des maltraitances dans l'enfance semble par conséquent avoir des effets spécifiques sur les systèmes de réponse subséquents qui connectent l'hypothalamus, l'hypophyse et les glandes surrénales. Cela entraîne une sensibilisation neurophysiologique précoce, engendrant d'intenses réponses aux stress futurs, 
en termes de production et réception de glucocorticoïdes (Bremner, 2005).

\section{La relation entre les expériences négatives de l'enfance et les maladies somatiques}

Les conséquences des ENE ne sont pas seulement représentées par des troubles cliniques spécifiques tels que l'état complexe de stress post-traumatique (ESPT-c), les troubles dissociatifs ou le trouble de la personnalité borderline (Liotti \& Farina, 2011) ; elles exercent leur influence sur un large éventail de maladies somatiques à l'âge adulte, y compris le cancer (Brown \& coll., 2010 ; Brown, Thacker \& Cohen, 2013 ; Fuller-Thompson \& Brennenstuhl, 2009). Wegman et Stetler (2009) ont procédé à une méta-analyse dans laquelle ils ont pris en compte les influences des maltraitances infantiles sur les résultats médicaux chez une population de jeunes adultes. Ils ont montré qu'il existait un lien important entre la présence de maltraitances dans l'enfance et les mauvais résultats physiques et médicaux à l'âge adulte.

Le système nerveux est fortement influencé par des événements stressants de type chronique et/ou aigu ; le système nerveux sympathique, l'axe HHS et le système adrénergique sont les trois grands systèmes particulièrement impliqués dans la réponse au stress neurobiologique. L'excitation, la réponse au stress, le comportement, la régulation émotionnelle et neuro-développementale sont tous reliés à eux (De Bellis, 2012). Le point essentiel est que l'axe HHS atteint son plein développement au cours des cinq premières années de l'enfance (Sapolsky \& Meaney, 1986), et il est par conséquent admis que des événements traumatiques ayant lieu pendant cette période sont néfastes pour le développement de l'enfant et que leurs effets à long terme peuvent traduire leurs conséquences épigénétiques. Ces dernières consistent en une altération permanente de l'expression génétique dans le système neuronal lié à la régulation de la réactivité aux facteurs de stress (Meaney \& Szyf, 2005). Dans ce contexte, les soins parentaux et donc le système d'attachement sont un facteur protecteur contre un développement neurobiologique inadapté (Fisher \& Gunnar, 2012).

Les expériences de vie négatives peuvent dérégler la fonction immunitaire cellulaire et les réponses autonomes, et conduire à des niveaux plus élevés du cortisol lié au stress (Dowd, Palermo \& Aiello, 2012 ; Fagundes, Glaser, Malarkey \& Kiecolt-Glaser, 2013 ; Shirtcliff, Coe \& Pollak, 2009). L'activation de l'axe HHS et l'hypercortisolémie subséquente opèrent souvent comme un mécanisme de réaction négative permettant le contrôle d'une réponse immunitaire ou inflammatoire amplifiée déclenchée par la chaîne des cytokines. Cet équilibre délicat semble faire partie d'un mécanisme homéostatique dont le dysfonctionnement pourrait entraîner des troubles graves tels qu'une infection, un cancer ou une maladie auto-immune. Dans la mesure où le cerveau joue un rôle central au sein de ce cycle, des facteurs tels que les émotions ou le stress d'origine psychosociale peuvent influencer négativement l'équilibre de ce mécanisme (Kronfol \& Remick, 2014). En outre, il a été démontré que les changements immunologiques liés au stress peuvent affaiblir la réponse des barrières naturelles contre le cancer ou les infections virales (Kawamura, Kim \& Azukai, 2001 ; Kiecolt-Glaser \& Glaser, 1999 ; Laudenslager et coll., 1998 ; Leonard \& Song, 1996 ; Nunnes et coll., 2002).

En 2010, Felitti et Anda ont avancé l'hypothèse que les ENE ont un impact direct sur l'installation du cancer et d'autres maladies graves. Les enfants ayant souffert d'événements traumatiques peuvent développer des comportements à risque pour leur santé (Hussey, Chang \& Kotch, 2006), des maladies physiques (p. ex. un trouble cardiaque ischémique, le diabète, le cancer, des maladies pulmonaires chroniques, des fractures osseuses, des maladies du foie), des problèmes sociaux et des déficiences dans leurs compétences sociales, émotionnelles et cognitives (Felitti, 2002 ; Felitti \& coll., 1998 ; Shapiro, 2014) au cours de leur vie.

On peut supposer qu'il existe deux types de causalités biologiques entre les ENE et le cancer : un " effet direct » et un " effet indirect ». L'effet direct inclut toutes les altérations biologiques causées par le stress lié à la maladie ; l'effet indirect, quant à lui, est perceptible dans des comportements susceptibles de mettre la santé du sujet en danger (p. ex. consommation de tabac, obésité sévère, dépression, promiscuité, tentatives de suicide, alcoolisme et toxicomanie ; Anda \& coll., 1999 ; Brown et coll., 2010 ; Delpierre \& Kelly-Irving, 2011 ; Kelly-Irving \& coll., 2013 ; Monahan \& Forgash, 2012 ; Shapiro, 2014). Dans une étude récente, Larkin, Felitti et Anda (2014) soulignent l'importance d'introduire une perspective biopsychosociale dans l'étude des expériences d'enfance difficiles.

Les ENE semblent créer une sorte de cycle vicieux entre des aspects psychosociaux et la santé physique, un circuit qui s'autoalimente : le premier nourrit négativement le second, et le second le premier, entraînant une augmentation graduelle des paramètres impliqués. Le but du traitement EMDR chez les patients 
adultes atteints de cancer est de " réparer les anciens souvenirs somatiques » et de briser ainsi ce cycle.

\section{La nature traumatique du cancer : un point de vue selon lequel l'événement du cancer est inscrit dans I'histoire de vie du patient}

Traumatisme signifie "blessure" en grec ( $\boldsymbol{\tau} \boldsymbol{\rho} \boldsymbol{\alpha} \boldsymbol{v} \boldsymbol{\mu} \boldsymbol{\alpha}$ : perforer, endommager, blesser, ruiner) et fait doublement référence à une plaie ouverte et aux répercussions d'un choc violent sur le corps entier. Le terme de trauma s'est largement répandu dans le domaine médico-chirurgical au cours du $18^{\mathrm{e}}$ siècle et a été repris par la psychiatrie et la psychologie clinique pour indiquer qu'un sujet est submergé par un excès de stimuli et dans l'incapacité d'accéder à ses défenses et de réagir (Van Der Kolk, McFarlane \& Weisaeth, 2005). La notion psychologique de trauma inclut l'ampleur, l'intensité et la précocité du trauma, de même que les aspects du tempérament, de la personnalité, du style d'attachement, de la sensibilité et de la résilience propres à un individu, ainsi que la présence ou l'absence d'un réseau de soutien social. Les réponses d'un sujet à un trauma peuvent aller de l'expérience d'émotions perturbantes et incontrôlées, le plus souvent négatives et douloureuses, à la désorientation et à un sentiment de perte de contrôle, avec des comportements dysfonctionnels, comme des défenses d'évitement ou de fuite. Ces réactions peuvent guérir ou disparaître spontanément après une courte période, tout en continuant à générer des symptômes liés à la personnalité du patient, qui sont malaisés à observer de l'extérieur. Dans les cas les plus difficiles, les patients peuvent développer un trouble dysfonctionnel chronique tel que l'ESPT (Manuel diagnostic et statistique des troubles mentaux [ $5^{\mathrm{e}}$ éd. ; DSM-5] ; American Psychiatric Association [APA], 2013). Devant un événement inacceptable, incompréhensible, terrifiant et insupportable, la capacité naturelle de la psyché humaine à le traiter peut être mise en péril.

La réponse individuelle au trauma dépend d'une variabilité subjective et intrasubjective, l'étape de vie dans laquelle la personne se trouve au moment du trauma, ses capacités à mentaliser, et la construction psychologique du «trauma » inclut des variables objectives (l'ampleur, l'intensité, le temps d'installation de l'événement) et subjectives (les traits de personnalité, le style d'attachement, la sensibilité, la résilience, le soutien social). Un trauma ne peut être défini uniquement par l'intensité objectivement produite par un événement extérieur stressant, mais nécessite également de prendre en considération des réponses subjectives internes qui dépendent du statut d'attachement et des ressources internes du patient. Les expériences traumatiques conduisent l'individu à présenter, tout au long de sa vie, des comportements liés à l'attachement à travers des modèles de travail internes, comme la recherche d'aide, de réconfort et de protection ; toutefois, des recherches récentes ont permis d'observer des différences liées à l'âge dans les comportements d'attachement et l'état d'esprit, principalement en relation avec les modifications des rôles sociaux au cours de la vie, mais également basées sur l'influence de ces rôles sur le développement de la personnalité (Chopik, Edelstein \& Fraley, 2013 ; Roberts, Wood \& Smith, 2005).

Le DSM-5 a introduit des changements importants dans la description des caractéristiques cliniques de l'ESPT par rapport au précédent manuel diagnostique et statistique des troubles mentaux ( $4^{e}$ éd., texte révisé ; DSM-IV-TR ; APA, 2000). Le DSM-5 ne considère plus l'ESPT comme un trouble anxieux : il l'intègre dans un nouveau chapitre appelé "Trauma, stress et troubles associés » et il se centre sur les symptômes comportementaux qui accompagnent l'ESPT. De plus, les critères comprenant des réponses subjectives comme une peur intense, un sentiment d'impuissance ou d'horreur, qui, dans le DSM-IV (APA, 1994) représentaient une innovation clinique par rapport au DSM-III (APA, 1980), ont également été supprimés.

En raison de la variabilité des résultats, on débat encore, aujourd'hui, du point de vue selon lequel les maladies mortelles peuvent provoquer des états traumatiques. Aujourd'hui, les diagnostics concordent rarement et il n'existe pas de ligne directrice commune concernant le diagnostic : plusieurs études sur l'ESPT dans des services de cancérologie soutiennent que les résultats psychologiques sont liés à l'ESPT (Kangas, Milross, Taylor \& Bryant, 2013 ; Posluszny, Edwards, Dew \& Baum, 2011), alors que d'autres considèrent ce point de vue comme surestimé (Menhert $\&$ Koch, 2007 ; Phipps \& coll., 2014). On a mis en cause la possibilité d'attribuer l'étiquette d'ESPT aux patients atteints du cancer, dans la mesure où la prévalence varie considérablement, révélant souvent qu'une minorité seulement des patients atteignent le seuil aux critères permettant le diagnostic d'ESPT (Kangas \& coll., 2002 ; Thompson, Eccleston \& Hickish, 2011). En outre, les symptômes d'ESPT sont susceptibles d'être confondus avec les conséquences secondaires de la maladie : la peur légitime d'une rechute peut rappeler des symptômes intrusifs (Hodges \& Humphris, 2009 ; Mehnert, Berg, Henrich \& Herschbach, 2009) et les effets secondaires de la chimiothérapie peuvent imiter les symptômes d'une hyperactivation (Kangas \& coll., 2002 ; Shelby, Golden-Kreutz \& Andersen, 2008 ; Thompson et coll., 2011). 
Diverses données semblent prouver que, bien que les classifications nosographiques du DSM et de la Classification internationale des maladies $\left(10^{\mathrm{e}}\right.$ révision, CIM-10) aient renforcé la fiabilité du diagnostic des symptômes dépressifs, ces manuels ne détectent pas précisément la nature et le spectre de variabilité des états psychologiques et de la souffrance sociale chez les patients atteints du cancer. L'existence d'un lien entre le cancer et l'EPST est appuyée par diverses recherches scientifiques qui indiquent que 3 à $35 \%$ des patients atteints du cancer développent un ESPT lié à la maladie (Kangas et coll., 2002).

Une des considérations cliniques les plus importantes est la façon dont le récit des expériences hautement traumatiques des patients peut être pris en charge, lorsque les critères du DSM- 5 ne sont pas pleinement remplis. Si les conditions entourant un événement remplissent les critères comprenant une menace imminente et objective sur la vie d'un individu (tremblements de terre, ouragans, inondations, situations extrêmement violentes), qu'en est-il lorsque la menace n'est pas extérieure, comme c'est le cas lorsqu'un patient s'entend annoncer un diagnostic de cancer mortel ? La définition d'un événement traumatique s'applique-t-elle uniquement aux circonstances prévues dans la classification du DSM-5 ou pouvons-nous nous fier aux récits douloureux de nos patients lorsqu'ils partagent avec nous, en thérapie, leur parcours avec la maladie?

En théorie, le cancer est capable d'être un événement traumatique dans la mesure où le diagnostic et le traitement qui s'ensuit représentent une véritable menace pour la vie et l'intégrité physique des patients. Un vaste corpus de littérature concernant la population souffrant du cancer appuie l'existence d'une forme spécifique d'ESPT, d'un ESPT lié au cancer (p. ex. Cordova, Studts, Hann, Jacobsen \& Andrykowsky, 2000 ; Smith \& coll., 2011; Thompson \& coll., 2011). En ce sens, le cancer est considéré comme « un processus de transformation perfide, incontrôlable et invasif conduisant graduellement à la mort " (Saccomani, 1998) partageant avec d'autres maladies graves plusieurs aspects traumatisants : c'est une menace pour l'intégrité physique et psychologique du patient ou d'un de ses proches (traumatisation secondaire), engendrant des sentiments de vulnérabilité, de perte de contrôle et d'impuissance. Les patients atteints d'un cancer peuvent avoir des réactions émotionnelles intenses dans leur fonctionnement quotidien, avec de hauts niveaux d'activation, des comportements d'évitement, ainsi que des pensées intrusives pouvant perturber leurs capacités normales de fonctionnement, pendant et après l'événement traumatique. De plus, les rechutes peuvent entraîner une retraumatisation. Parmi les nombreux facteurs subjectifs de risque d'apparition d'une maladie organique, les recherches ont identifié deux situations principales : le sentiment d'impuissance et le désespoir (Schmale \& Engel, 1967) qui semblent découler de la réponse émotionnelle de la personne aux séparations ou aux pertes affectives. Schmale \& Engel (1967) estiment que ces événements stressants, couplés avec des états de désespoir et d'abandon de l'environnement social, mènent à une plus grande vulnérabilité et peuvent déclencher une maladie. Les sensations d'impuissance et de désespoir, chez les patients atteints du cancer, réduisent significativement leur qualité de vie (GustavssonLilius, Julkunen \& Hietanen, 2007) et sont associées à un désir fort de mourir plus vite, à des demandes de suicide assisté et d'euthanasie (Rodin $\&$ coll., 2007), à un taux de rechute plus élevé et à des pronostics plus sombres (Watson, Haviland, Greer, Davidson \& Bliss, 1999 ; Watson, Homewood, Haviland \& Bliss, 2005).

Nous pensons que les phénomènes factuels observables, comme les données recueillies à partir d'une évaluation de la gravité de la maladie, l'existence d'un réel risque de mort, les interventions chirurgicales, ont un effet moindre que la subjectivité de l'expérience (Ganz et coll., 1993 ; Härtl et coll., 2010 ; Maunsell, Brisson, Dubois, Lauzier \& Fraser 1999 ; Rowland et coll., 2000). De plus, il y a certaines caractéristiques qui différencient le cancer d'autres événements traumatiques et le rendent difficile à situer dans le champ post-traumatique : d'abord, contrairement à tout autre événement préjudiciable à la santé, le cancer est complexe et durable, et c'est davantage un vécu de traumas de durée indéfinie et qui se répètent au cours du temps qu'un événement aigu précis (Borio \& Torta, 2007). Cela rend l'ESPT d'un patient atteint du cancer très différent du même trouble chez quelqu'un ayant vécu une seule expérience traumatique (Greimel et coll., 2013). Ensuite, la menace intrinsèque que représente le cancer est par nature anticipatoire, dans la mesure où les pensées intrusives sont orientées vers les conséquences futures de la maladie, telles que la peur de la rechute (Mehnert et coll., 2009). Enfin, contrairement à un événement traumatique précis dont les origines sont extérieures à l'individu (p. ex. agression, catastrophes naturelles, accidents aériens), la menace du cancer possède une importante composante interne, surtout sur le plan d'une impression d'inéluctabilité (Gurevich, Devins \& Rodin, 2002). Compte tenu de la discussion ci-dessus, il semble important de réfléchir à la pertinence de parler de stress post-traumatique à propos du cancer, dont personne n’a jamais été déclaré " définitivement guéri » (Trotti \& Bellani, 2010). 
D’après la littérature scientifique, les preuves semblent aller dans le sens de la présence plus fréquente d'un état de stress post-traumatique (ESPT) ou d'un ESPT subclinique que d'un ESPT proprement dit. Les groupes de symptômes semblent être en dessous du seuil selon la classification du DSM, mais ils représentent néanmoins un état fortement invalidant pour les patients. Gurevich et coll. (2002) ont rapporté que seulement $3 \%$ à $4 \%$ des patients ayant récemment reçu un diagnostic de cancer présentaient un véritable ESPT, alors que $20 \%$ présentaient des symptômes en dessous du seuil diagnostique. De plus, $35 \%$ des patients présentaient un trouble en dessous du seuil après leur traitement actif, alors que $80 \%$ présentaient ce trouble lors d'une rechute de la maladie. Ces pourcentages sont importants et suggèrent la nécessité d’identifier les symptômes-clés permettant d'évaluer l'ESPT situé en dessous du seuil diagnostique (Blanchard, Jones-Alexander, Buckley $\&$ Forneris, 1996 ; Marshall et coll., 2001 ; Stein, Walker, Hazen \& Forde, 1997). Ces résultats donnent à penser que les réactions au stress d'origine traumatique doivent être conceptualisées sur un continuum. Les auteurs suggèrent d'abandonner un modèle catégorique pour identifier avec plus de précision les cas qui requièrent une intervention clinique. Les symptômes intrusifs liés à des souvenirs perturbants et la reviviscence d'un trauma lié au diagnostic du cancer semblent dominants (Matsuoka et coll., 2006) et s'expriment chez les patients à travers des inquiétudes constantes, la peur de la rechute, des cauchemars, des flashbacks et une confiance réduite en l'avenir. Ces souvenirs intrusifs sont reliés à des comportements d'adaptation inappropriés, des comportements d'évitement et des déficits de la mémoire autobiographique (Brewin et coll., 1998) qui jouent un rôle important dans le fonctionnement du patient et peuvent entraver le processus d'acceptation de la maladie (Whitaker, Brewin $\&$ Watson, 2008). En outre, des recherches récentes menées par Civilotti et coll. (2015) ont avancé l'hypothèse d'une réaction psychologique spécifique de dissociation, qui peut être attribuée au spectre traumatique dans le contexte du cancer.

Les techniques modernes de neuro-imagerie permettent d'observer comment, chez les patients atteints du cancer, les symptômes post-traumatiques, et en particulier les symptômes intrusifs, activent les mêmes régions cérébrales que celles observées dans les études sur l'ESPT. À l'instar des individus atteints d'ESPT, les patients souffrant d'un cancer montrent une réduction importante du volume del'hippocampe, la zone du cerveau impliquée dans le traitement des souvenirs autobiographiques (Nakano et coll., 2002 ;
Shin, Rauch \& Pitman, 2006). Des patients atteints du cancer et souffrant de souvenirs intrusifs présentaient des réductions notables du volume de l'amygdale (Matsuoka, Yamawaki, Inagaki, Akechi \& Uchitomi, 2003). Les mêmes données ont été découvertes chez des patients cancéreux souffrant d'une dépression liée à leur maladie, comparés à des patients qui ne développaient pas la même symptomatologie (Yoshikawa et coll., 2006). Dans le cortex orbitofrontal, les patients atteints du cancer présentant des symptômes posttraumatiques montrent également une altération structurelle qu'on ne retrouve pas chez des patients ne présentant pas ces symptômes (Hakamata et coll., 2007). D’autres observations de patients ayant reçu un diagnostic de cancer révèlent des modifications structurelles et fonctionnelles de régions spécifiques et de certains circuits neuronaux (Kandel, Schwartz \& Jessell, 2000), rendant compte de la propriété plastique intrinsèque permettant au cerveau humain d'adapter ses fonctions et d'opérer des changements en fonction de l'expérience du sujet.

\section{Le rôle de la thérapie EMDR en psycho-oncologie}

Les expériences nouvelles, telles que le diagnostic du cancer, forment un flux sans fin d'informations, conscientes et inconscientes, et les données sensorielles, cognitives, émotionnelles et somatiques qui s'y rapportent sont enregistrées dans des réseaux de souvenirs en vue d'applications futures, orientant les réactions de la personne vers son environnement. Dans des conditions normales, ce système est adapté, mais lorsque des événements négatifs perturbants se produisent, le système de traitement de l'information peut se trouver submergé et n'est alors plus capable d'assimilation. En particulier, lorsque ces événements se répètent quotidiennement, les informations liées à l'événement négatif sont enregistrées à part, et sont dès lors incapables de se relier à des réseaux de souvenirs plus adaptés. Certaines situations du présent peuvent parfois réactiver ces souvenirs, entraînant des comportements inadaptés ou symptomatiques. L’EMDR intervient précisément sur les événements traumatiques non traités (Faretta, Callerame \& Civilotti, 2014).

Le travail clinique sur le passé lointain d'un patient doit étudier son histoire développementale, avec une attention particulière portée à l'attachement qu'il a connu. Dans le cas qui nous intéresse, il semble important d'investiguer un possible état de vulnérabilité attribuable à des causes psychogènes, comme la présence de traumas connectés à l'activation du 
système d'attachement dans l'histoire personnelle du développement.

Outre cette investigation sur les vécus traumatiques imputables à l'histoire développementale du patient, il semble également nécessaire de centrer le travail clinique sur la dimension temporelle du passé récent et d'aborder l'expérience traumatique du patient en tant que malade. Il semble également important de centrer le travail sur sa situation de vie actuelle, dans une dimension présente qui s'étend dans le futur. Comme dans toutes les autres dimensions temporelles mentionnées plus haut, l'influence du système d'attachement social est importante, en particulier en ce qui concerne la façon dont les émotions sont régulées chez le patient, sa perception du soutien social, sa capacité à demander de l'aide et, en conséquence, les différentes stratégies d'adaptation qu'il adoptera, son adaptation psychologique à la maladie, et sa survie (Faretta, 2014).

\section{Les recherches EMDR en psycho-oncologie et avec les affections médicales}

Au cours des vingt dernières années, de nombreuses études ont reconnu l'EMDR comme un traitement fondé sur des bases factuelles et efficace pour le traitement du trauma dans les directives cliniques de nombreuses organisations professionnelles et de nombreux services nationaux de santé mentale. En 2000, l'EMDR a été incluse dans les recommandations de la Société internationale pour les recherches sur les stress traumatiques (ISTSS) en tant qu'approche thérapeutique fondée sur des bases factuelles, et en 2004, l'APA a reconnu son efficacité en l'incluant parmi ses directives de pratique clinique (Faretta, 2014, 2015).

Depuis 2000, l'EMDR a obtenu une reconnaissance internationale, montrant son efficacité clinique. Différents essais ont établi qu'il était efficace d'utiliser l'EMDR en psychologie sur une plus large gamme de troubles. De nombreuses méta-analyses (Bisson $\&$ Andrew, 2007) montrent que l'EMDR produit des effets thérapeutiques similaires à d'autres méthodes qui ont fait l'objet de plus de recherches scientifiques, comme la thérapie cognitive comportementale (TCC).

Dans le domaine de la psycho-oncologie, la première étude contrôlée portant sur le traitement de patients atteints du cancer a été conduite par Capezzani et coll. (2013). Cette étude soulignait l'efficacité majeure de l'EMDR par rapport à la TCC dans le traitement de l'ESPT de patients en oncologie, lors de la phase de suivi de la maladie. Après traitement, les patients traités avec l'EMDR souffraient significativement moins d’un ESPT que ceux traités avec la TCC. Les résultats à l'Impact of Event Scale-Revised à (IES-R) et à la Clinician-Administered PTSD Scale (CAPS) avaient baissé significativement dans le groupe EMDR par rapport au groupe TCC. L'EMDR a montré la même efficacité lors de la phase de traitement actif du cancer que dans les phases de suivi. L'anxiété et la dépression, cependant, s'étaient améliorées de façon identique dans les deux groupes de traitements.

En 2014, Faretta et coll. ont présenté une étude clinique pilote sur 18 patients atteints du cancer. Les résultats préliminaires montraient qu'après 12 séances d'EMDR, on observait une atténuation de diverses formes de complications psychologiques dues à l'impact du diagnostic du cancer, en particulier une rémission des symptômes d'ESPT, une diminution des niveaux d'anxiété, de dépression et de perception de la douleur. Une étude pilote conduite par Jarero et coll. (2015) a évalué l'efficacité du protocole EMDR de traitement intégratif de groupe (EMDR Integrative Group Treatment Protocol-EMDR-PTIG) spécifiquement pour des femmes atteintes de différents types de cancer et les symptômes d'ESPT associés qu'elles présentaient. Les résultats statistiques montrèrent une amélioration importante, tant dans la phase active que dans la phase de suivi du cancer. De plus, suite au traitement EMDRPTIG, les patientes faisaient état d'une amélioration générale. Il est clair que d'autres études contrôlées randomisées sont nécessaires pour démontrer l'efficacité de l'EMDR-PTIG sur cette population, mais les résultats positifs de cette étude pilote suggèrent que ce protocole peut être un soutien valable pour des patients atteints de cancer et souffrant de symptômes d'ESPT.

Une étude clinique comparative sur des patients en radiothérapie, conduite récemment en Iran par Majidzadeh et Sediq (2015a, 2015 b) avec un plan simple de recherche prétest/post-test, semble également confirmer l'efficacité de l'EMDR dans la gestion du stress, de l'anxiété et de la dépression chez des patients atteints de cancer. Comme la méthodologie de cette étude n'est pas bien exposée, nous interprétons ces résultats comme un indicateur positif, mais sans la nécessaire précision d'une étude scientifique contrôlée. En 2002, Peters, Wissing et Du Plessis ont rapporté les résultats d'une étude descriptive portant sur des cas multiples où trois patients atteints du cancer avaient été traités en EMDR et trois autres par une thérapie de soutien. Dans cette étude, le recueil des données était triangulé en termes de résultats cliniques quantitatifs, d'entretiens semi-structurés et par un entretien conduit par un chercheur extérieur. Les résultats, d'un point de vue objectif et subjectif, allaient en faveur de l'EMDR. 
L’EMDR montre également son efficacité dans d'autres contextes cliniques. Par exemple, des patients qui ont survécu à un accident cardiaque peuvent développer des symptômes d'ESPT et des symptômes anxieux et dépressifs associés. Dans ces cas, le thérapie EMDR s'est avéré très efficace (Arabia, Manca \& Salomon, 2011). Les résultats de cette étude pilote apportent un soutien préliminaire à l'efficacité de l'EMDR auprès des victimes d'accidents cardiaques. Behnam Moghadam, Behnam Moghadam \& Salehian (2015) concluent que l'EMDR est une méthode efficace pour traiter et réduire la dépression chez des patients ayant un infarctus du myocarde, et qu'elle peut donc être utilisée efficacement pour le traitement de patients en unités de soins intensifs.

En 2002, Grant et Threlfo ont mis en lumière l'efficacité du protocole EMDR pour la douleur chronique, pour réduire les niveaux de douleur et leurs effets négatifs. Dans leur étude, Mazzola et coll. (2009) ont étudié les effets de l'EMDR sur 38 patients souffrant de douleurs chroniques (en particulier des maux de tête) avec 12 séances hebdomadaires de 90 minutes ; confirmant leur hypothèse, l'EMDR a modifié les réactions émotionnelles à la douleur. Une récente revue systématique de la littérature clinique (Tesarz et coll., 2014) a de nouveau souligné l'efficacité du traitement EMDR pour soulager les douleurs chroniques. Il est donc recommandé de renforcer cette zone de recherche. Dans la mesure où les douleurs chroniques sont communes chez les patients atteints du cancer (Green, Hart-Johnson \& Loeffler, 2011), l'EMDR pourrait être une intervention intéressante pour cette seule raison déjà.

Une étude de 2007 de Schneider, Hofmann, Rost \& Shapiro décrit l'application du modèle TAI de Shapiro dans le traitement des douleurs de membres fantômes (DMF), et en 2008, les mêmes chercheurs ont mené une étude qui confirme l'influence positive de l'EMDR sur la diminution ou l'élimination de la douleur fantôme, influence confirmée lors du suivi à long terme. L'étude a souligné une réduction de la dépression et des symptômes d'ESPT ainsi qu'une réduction importante ou même l'élimination du traitement médicamenteux que prenait le patient pour sa douleur fantôme. En 2013, une étude conduite par Amano, Seiyama et Toichi, au Japon, sur une patiente souffrant de DMF, a démontré l'efficacité du protocole DMF de l'EMDR. Il est possible d'en déduire l'implication d'un mécanisme semblable à l'ESPT dans la pathogenèse de la DMF, de même que l'efficacité de l'EMDR dans le traitement de la DMF. Une récente étude de cas publiée par Brennstuhl et coll. (2015) avec deux patientes atteintes d'un syndrome de sein fantôme montre l'efficacité de la thérapie EMDR sur les résultats qualitatifs et quantitatifs et le changement clinique obtenu en produisant une modification de la représentation du corps. Des résultats positifs ont également été observés dans le traitement de crises psychogènes (Chemali $\&$ Meadows, 2004) et de crises psychogènes non épileptiques (Kelley $\&$ Benbadis, 2007), toutes deux présentant une comorbidité avec un ESPT, de même que dans des cas d'épilepsie et de déficience intellectuelle légère (Rodenburg, Benjamin, Meijer \& Jongeneel, 2009).

\section{L'EMDR chez les patients atteints de cancer : directions actuelles et futures}

La vision holistique de la maladie reconnaît la connexion existant entre l'esprit et le corps et emploie une approche de traitement qui prend en compte la complexité du système, avec une attention particulière portée à leurs influences réciproques. La maladie physique peut causer une grande douleur psychologique. De même, l'esprit peut influencer des processus physiologiques et intervenir sur les circuits de modulation endocriniens en réponse aux facteurs de stress.

Une revue récente des études publiées (Shapiro, 2014), des recherches sur les ENE (Felitti et coll., 1998), de même qu'un grand nombre d'études scientifiques conduites en psychologie et en biomédecine révèlent comment les expériences de vie difficiles engendrent le développement de maladies et conduisent finalement à des états chroniques. De nombreux symptômes post-traumatiques proviennent des difficultés cognitives de la personne à faire face à un événement traumatique et de son échec à retraiter les cognitions traumatiques liées à ce souvenir.

L'intervention EMDR doit par conséquent prendre en considération certains aspects. Tout d'abord, l'ajustement psychologique à la maladie est influencé par des caractéristiques personnelles relativement stables (statut social, personnalité, expériences de vie), par des variables qui peuvent influencer l'ajustement global de l'individu à la maladie et sont susceptibles de changer au fil du temps (stratégies d'adaptation), ainsi que par le traitement et les techniques thérapeutiques employés (De Picolli, 2014 ; Morasso, Di Leo \& Grassi, 2002 ; Zani \& Cicognani, 1999).

La spécificité de la psycho-oncologie consiste à aborder un patient dont le malaise psychologique ne provient pas en premier lieu d'un trouble psychopathologique, mais de la situation traumatisante liée à la maladie (Morasso \& coll., 2002). L'intervention psychothérapique comporte donc les objectifs suivants (Faretta, 2014 ; Varetto, Ramonda, Stanizzo, \& 
Torta, 2007) : (a) limiter la souffrance, encourager le patient à verbaliser ses pensées et sentiments négatifs ; (b) aider le patient à développer des comportements mieux adaptés, lui restituant une maîtrise sur sa vie ; (c) favoriser la communication entre le patient, le personnel médical et la famille, permettant la résolution des difficultés pratiques liées au traitement médical ; (d) éclairer le patient sur l'influence des facteurs psychologiques sur le cancer; et (e) rendre le sentiment d'un avenir possible au patient et à sa famille.

Étant donné ces conditions, et à la lumière des points esquissés dans le présent article, on peut affirmer qu'une intervention clinique avec l'EMDR en psycho-oncologie passe obligatoirement par plusieurs niveaux de travail, associés aux diverses dimensions temporelles de la vie du patient. Le modèle TAI fournit un cadre théorique exhaustif et cliniquement fécond, guidant le démantèlement des circuits qui alimentent les réponses émotionnelles pathologiques et perpétuent de hauts niveaux de souffrance, accompagnés de détresse physique et psychologique.

Le retraitement EMDR permet de changer les perceptions émotionnelles et physiques liées aux souvenirs traumatiques figés, ce qui permet l'intégration et la reconsolidation des éléments les plus perturbants, liés à la maladie ou provenant d'expériences passées douloureuses. Grâce à des protocoles conçus spécifiquement pour des patients atteints du cancer ou d'autres maladies organiques (Faretta, 2014, 2015), l'EMDR semble répondre avec succès au besoin de programmes thérapeutiques structurés qui prennent en compte, de façon holistique, les aspects physiques, corporels et sémantiques de l'expérience du patient. Premièrement, l'EMDR permet de guérir les blessures psychologiques résultant d'ENE et ensuite, grâce à l'utilisation du protocole pour le passé, le présent et le futur, aide le patient à gérer l'aspect physique et le timing imprévisible de la maladie.

Les recherches mentionnées plus haut sont encourageantes et suggèrent que l'approche EMDR peut être une thérapie efficace pour aider les patients atteints du cancer. Cependant, il n'existe qu'une étude contrôlée randomisée dans le scénario scientifique qui soutient l'utilisation de l'EMDR (Capezzani et coll., 2013), alors que le plan de recherche d'autres études reste parfois imprécis. Il est nécessaire de mener d'autres recherches, couvrant une plus large population de patients atteints de cancer, avec des groupes de contrôle pour comparer différents types d'approches cliniques, utilisant des plans de recherche randomisés et produisant davantage de preuves de résultats neurobiologiques, afin d'attester de l'efficacité de l'EMDR. En parallèle, il est essentiel d'intégrer les nouvelles recherches en psychologie, en neurobiologie et en médecine au panel de compétences et de stratégies cliniques employées dans la prise en charge des patients, en particulier en présence d'une comorbidité de symptômes physiques et psychologiques.

\section{Bibliographie}

Amano, T., Seiyama, A., \& Toichi, M. (2013). Brain activity measured with near-infrared spectroscopy during EMDR treatment of phantom limb pain. Journal of EMDR Practice and Research, 7 (3), 144-153.

American Psychiatric Association. (1980). Diagnostic and statistical manual of mental disorders ( $3^{\text {rd }}$ ed.). Washington, DC: Author.

American Psychiatric Association. (1994). Diagnostic and statistical manual of mental disorders ( $4^{\text {th }}$ ed.). Washington, DC: Author.

American Psychiatric Association. (2000). Diagnostic and statistical manual of mental disorders ( $4^{\text {th }}$ ed., text rev). Washington, DC: Author.

American Psychiatric Association. (2013). Diagnostic and statistical manual of mental disorders ( $5^{\text {th }}$ ed.), Washington, DC: Author.

Anda, R. F., Croft, J. B., Felitti, V. J., Nordenberg, D., Giles, W. H., Williamson, D. F., \& Giovino, G. A. (1999). Adverse childhood experiences and smoking during adolescence and adulthood. JAMA, 282(17), 1652-1658.

Anda, R. F., Felitti, V. J., Bremner, J. D., Walker, J. D., Whitfield, C., Perry, B. D., . . Giles, W. H. (2006). The enduring effects of abuse and related adverse experiences in childhood. A convergence of evidence from neurobiology and epidemiology. European Archives of Psychiatry and Clinical Neuroscience, 256, 174-186.

Arabia, E., Manca, M. L., \& Solomon, R. M. (2011). EMDR for survivors of life-threatening cardiac events: Results of a pilot study. Journal of EMDR Practice and Research, 5(1), 2-13.

Baider, L., \& Kaplan De-Nour, A. (1997). Psychological distress and intrusive thoughts in cancer patients. The Journal of Nervous and Mental Disease, 185, 346-348.

Behnam Moghadam, M., Behnam Moghadam, A., \& Salehian, T. (2015). Efficacy of eye movement desensitization and reprocessing (EMDR) on depression in patients with myocardial infarction (MI) in a 12-month follow up. Iranian Journal of Critical Care Nursing, 7 (4), 221-226.

Benedetti, F., Radaelli, D., Poletti, S., Falini, A., Cavallaro, R., Dallaspezia, S., . . Smeraldi, E. (2011). Emotional reactivity in chronic schizophrenia: Structural and functional brain correlates and the influence of adverse childhood experiences. Psychological Medicine, 41, 509-519.

Bisson, J., \& Andrew, M. (2007). Psychological treatment of post-traumatic stress disorder (PTSD). Cochrane Database of Systematic Reviews, (3) CD003388.

Blanchard, E. B., Jones-Alexander, J., Buckley, T. C., \& Forneris, C. A. (1996). Psychometric properties of the 
PTSD Checklist (PCL). Behaviour Research and Therapy, 34, 669-673.

Borio, R., \& Torta, R. (2007). I disturbi psichici in oncologia [Mental disorders in oncology]. Psiconcologia. Torino: Centro Scientifico Editore, 58-62.

Bowlby, J. (1988). A secure base. London, United Kingdom: Routledge.

Boyce, W. T., \& Ellis, B. J. (2005). Biological sensitivity to context: I. An evolutionary-developmental theory of the origins and functions of stress reactivity. Development and Psychopathology, 17(2), 271-301.

Bremner, J. D. (2005). Effects of traumatic stress on brain structure and function: Relevance to early responses to trauma. Journal of Trauma \& Dissociation, 6(2), 51-68.

Brennstuhl, M. J., Tarquinio, C., Montel, S., Masson, J., Bassan, F., \& Tarquinio, P. (2015). Using eye movement desensitization and reprocessing (EMDR) as a treatment for phantom breast syndrome: Case study. Sexologies, 24(2), e29-e36.

Brewin, C. R., Watson, M., McCarthy, S., Hyman, P., \& Dayson, D. (1998). Intrusive memories and depression in cancer patients. Behaviour Research and Therapy, 36(12), 1131-1142.

Brown, D. W., Anda, R. F., Felitti, V. J., Edwards, V. J., Malarcher, A. M., Croft, J. B., \& Giles, W. H. (2010). Adverse childhood experiences are associated with the risk of lung cancer: A prospective cohort study. BMC Public Health, 10, 20.

Brown, M. J., Thacker, L. R., \& Cohen, S. A. (2013). Association between adverse childhood experiences and diagnosis of cancer. Plos One, 8 (6), e65524.

Bultz, B. D., \& Holland, J. C. (2006). Emotional distress in patients with cancer: The sixth vital sign. Community Oncology, 3, 311-314.

Bury, M. (1982). Chronic illness as biographical disruption. Sociology of Health \& Illness, 4(2), 167-182.

Capezzani, L., Ostacoli, L., Cavallo, M., Carletto, S., Fernandez, I., Solomon, R., . . . Cantelmi, T. (2013). EMDR and CBT for cancer patients: Comparative study of effects on PTSD, anxiety, and depression. Journal of EMDR Practice and Research, 7(3), 134-143.

Carrion, V. G., \& Wong, S. S. (2012). Can traumatic stress alter the brain? Understanding the implications of early trauma on brain development and learning. Journal of Adolescent Health, 51(2), S23-S28.

Chemali, Z., \& Meadows, M. E. (2004). The use of eye movement desensitization and reprocessing in the treatment of psychogenic seizures. Epilepsy \& Behavior, 5(5), 784-787.

Chopik, W. J., Edelstein, R. S., \& Fraley, R. C. (2013). From the cradle to the grave: Age differences in attachment from early adulthood to old age. Journal of Personality, 81(2), 171-183.

Civilotti, C., Castelli, L., Binaschi, L., Cussino, M., Tesio, V., Di Fini, G., . . . Torta, R. (2015). Dissociative symptomatology in cancer patients. Frontiers in Psychology, 6, 1-7.

Cordova, M. J., Studts, J. L., Hann, D. M., Jacobsen, P. B., \& Andrykowski, M. A. (2000). Symptom structure of PTSD following breast cancer. Journal of Traumatic Stress, 13(2), 301-319.

Cotraccia, A. J. (2012). Adaptive information processing and a systemic biopsychosocial model. Journal of EMDR Practice and Research, 6(1), 27-36.

Danese, A., \& McEwen, B. S. (2012). Adverse childhood experiences, allostasis, allostatic load, and age-related disease. Physiology \& Behavior, 106(1), 29-39.

De Bellis, M. D. (2012). Neurobiologia della trascuratezza infantile. In R. A. Lanius, E. Vermetten, \& C. Pain (Eds.), L'impatto del trauma infantile sulla salute e sulla malattia. L'epidemia nascosta [The impact of early life trauma on health and issue] (pp. 208-225). Rome, Italy: Giovanni Fioriti Editore.

Delpierre, C., \& Kelly-Irving, M. (2011). To what extent are biological pathways useful when aiming to reduce social inequalities in cancer? European Journal of Public Health, 21(4), 398-399.

De Piccoli, N. (2014). Salute e qualità della vita nella società del benessere. Prospettive interdisciplinari. Rome, Italie: Carocci Editore.

Dowd, J. B., Palermo, T. M., \& Aiello, A. E. (2012). Family poverty is associated with cytomegalovirus antibody titers in U.S. children. Health Psychology, 31(1), 5-10.

Drageset, S., Lindstrøm, T. C., Giske, T., \& Underlid, K. (2011). Being in suspense: Women's experiences awaiting breast cancer surgery. Journal of Advanced Nursing, 67(9), 1941-1951.

Engel, G. L. (1977). The need for a new medical model: A challenge for biomedicine. Science, 196(4286), 129-136.

Fagundes, C. P., Glaser, R., Malarkey, W. B., \& Kiecolt-Glaser, J. K. (2013). Childhood adversity and herpes virus latency in breast cancer survivors. Health Psychology, 32(3), 337-344.

Fallowfield, L., Ratcliffe, D., Jenkins, V., \& Saul, J. (2001). Psychiatric morbidity and its recognition by doctors in patients with cancer. British Journal of Cancer, 84, 1011-1015.

Faretta, E. (2014). Trauma e malattia: EMDR in psiconcologia [Trauma and illness: EMDR in psycho-oncology]. Milan, Italy: Mimesis Edizioni.

Faretta, E. (2015). Trauma and illness: EMDR in psycho-oncology. Milan, Italy: Mimesis Edizioni.

Faretta, E., Agazzi, T., Poli, E., Sacchezin, S., \& Zambon, V. (2013). EMDR in psiconcologia. Dalle memorie traumatiche all'attivazione delle risorse personali. Paper presented at the United Nations EMDR European Congress. Geneva, Switzerland.

Faretta, E., Callerame, C., \& Civilotti, C. (2014). EMDR in psiconcologia. Studio pilota per valutare l'efficacia dell'intervento EMDR in un campione di pazienti oncologici. In E. Faretta (Ed.), Trauma e malattia : EMDR in psiconcologia [Trauma and illness: EMDR in psychooncology] (pp. 244-252), Milan, Italy : Mimesis Edizioni.

Faretta, E., \& Sacchezin, S. (2015). Il contributo dell'approccio EMDR in psiconcologia: Un' integrazione tra mente e corpo. In B. Rossi (Ed.), Il gruppo tra mente e corpo. Percorsi terapeutici. Milan, Italy: Franco Angeli. 
Felitti, V. J. (2002). The relation between adverse childhood experiences and adult health: Turning gold into lead. The Permanente Journal, 6(1), 44-47.

Felitti, V. J., \& Anda, R. F. (2010). The relationship of adverse childhood experiences to adult medical disease, psychiatric disorders, and sexual behavior: Implications for healthcare. In R. Lanius, E. Vermetten, \& C. Pain (Eds.), The impact of early life trauma on health and disease: The hidden epidemic (pp. 77-87). Cambridge, United Kingdom: Cambridge University Press.

Felitti, V. J., Anda, R. F., Nordenberg, D., Williamson, D. F., Spitz, A. M., Edwards, V., . . . Marks, J. S. (1998). Relationship of childhood abuse and household dysfunction to many of the leading causes of death in adults. The adverse childhood experiences (ACE) study. American Journal of Preventive Medicine, 14(4), 245-258.

Fisher, P. A., \& Gunnar, M. (2012). Lo stress come fattore di rischio di malattia in età adulta. In R. A. Lanius, E. Vermetten, \& C. Pain (Eds.), L'impatto del trauma infantile sulla salute e sulla malattia. L'epidemia nascosta [The impact of early life trauma on health and issue] (pp. 226-240). Rome, Italy: Giovanni Fioriti Editore.

Fuller-Thomson, E., \& Brennenstuhl, S. (2009). Making a link between childhood physical abuse and cancer: Results from a regional representative survey. Cancer, 115(14), 3341-3350.

Ganz, P. A., Hirji, K., Sim, M. S., Schag, C. A., Fred, C., \& Polinsky, M. L. (1993). Predicting psychosocial risk in patients with breast cancer. Medical Care, 31(5), 419-431.

Granieri, A., Tamburello, S., Tamburello, A., Casale, S., Cont, C., Guglielmucci, F., \& Innamorati, M. (2013). Quality of life and personality traits in patients with malignant pleural mesothelioma and their first-degree caregivers. Neuropsychiatric Disease and Treatment, 9, 1193-1202.

Grant, M., \& Threlfo, C. (2002). EMDR in the treatment of chronic pain. Journal of Clinical Psychology, 58(12), 1505-1520.

Grassi, L., Biondi, M., \& Costantini, A. (2009). Manuale pratico di psico-oncologia. Rome, Italy: Il Pensiero Scientifico.

Green, C. R., Hart-Johnson, T., \& Loeffler, D. R. (2011). Cancer-related chronic pain: Examining quality of life in diverse cancer survivors. Cancer, 117(9), 1994-2003.

Greimel, E., Dorfer, M., Lambauer, M., Bjelic-Radisic, V., Gramm, S., Lahousen, M., \& Lang, U. (2013). Posttraumatic stress disorder in female cancer patients: An inappropriate diagnosis in oncology? Psychotherapy and Psychosomatics, 82(4), 271-272.

Gurevich, M., Devins, G. M., \& Rodin, G. M. (2002). Stress response syndromes and cancer: Conceptual and assessment issues. Psychosomatics, 43(4): 259-281.

Gustavsson-Lilius, M., Julkunen, J., \& Hietanen, P. (2007). Quality of life in cancer patients: The role of optimism, hopelessness, and partner support. Quality of Life Research, 16(1), 75-87.

Hakamata, Y., Matsuoka, Y., Inagaki, M., Nagamine, M., Hara, E., Imoto, S., . . . Uchitomi, Y. (2007). Structure of orbitofrontal cortex and its longitudinal course in cancer-related post-traumatic stress disorder. Neuroscience Research, 59(4), 383-389.

Härtl, K., Engel, J., Herschbach, P., Reinecker, H., Sommer, H., \& Friese, K. (2010). Personality traits and psychosocial stress: Quality of life over 2 years following breast cancer diagnosis and psychological impact factors. Psycho-Oncology, 19(2), 160-169.

Hodges, L. J., \& Humphris, G. M. (2009). Fear of recurrence and psychological distress in head and neck cancer patients and their carers. Psycho-Oncology, 18(8), 841-848.

Holland, J. C. (1989). Now we tell — but how well? Journal of Clinical Oncology, 7, 557-559.

Holland, J. C. (2004). IPOS Sutherland memorial lecture: An international perspective on the development of psychosocial oncology: Overcoming cultural and attitudinal barriers to improve psychosocial care. PsychoOncology, 13(7), 445-459.

Holzner, B., Giesinger, J., \& Efficace, F. (2014). L'importanza della valutazione della qualità della vita nei pazienti oncologici. In T. N. Wise, M. Biondi, \& A. Costantini (Eds.), Psiconcologia. Milan, Italy: Cortina Raffaello.

Hou, W. K., \& Lam, J. H. (2014). Resilience in the year after cancer diagnosis: A cross-lagged panel analysis of the reciprocity between psychological distress and wellbeing. Journal of Behavioral Medicine, 37(3), 391-401.

Hussey, J. M., Chang, J. J., \& Kotch, J. B. (2006). Child maltreatment in the United States: Prevalence, risk factors, and adolescent health consequences. Pediatrics, 118(3), 933-942.

Jarero, I., Artigas, L., Uribe, S., García, L. E., Cavazos, M. A., \& Givaudan, M. (2015). Pilot research study on the provision of the EMDR integrative group treatment protocol with female cancer patients. Journal of EMDR Practice and Research, 9(2), 98-105.

Kandel, E. R., Schwartz, J. H., \& Jessell, T. M. (2000). Principles of neural science (4th ed.). New York, NY: McGraw-Hill.

Kangas, M., Henry, J. L., \& Bryant, R. A. (2002). Posttraumatic stress disorder following cancer. A conceptual and empirical review. Clinical Psychology Review, 22(4), 499-524.

Kangas, M., Milross, C., Taylor, A., \& Bryant, R. A. (2013). A pilot randomized controlled trial of a brief early intervention for reducing posttraumatic stress disorder, anxiety, and depressive symptoms in newly diagnosed head and neck cancer patients. Psycho-Oncology, 22(7), $1665-1673$.

Kawamura, N., Kim, Y., \& Asukai, N. (2001). Suppression of cellular immunity in men with a past history of posttraumatic stress disorder. American Journal of Psychiatry, 158(3), 484-486.

Kelley, S. D. M., \& Benbadis, S. (2007). Eye movement desensitization and reprocessing in the psychological treatment of trauma-based psychogenic non-epileptic seizures. Clinical Psychology \& Psychotherapy, 14(2), $135-144$ 
Kelly-Irving, M., Lepage, B., Dedieu, D., Lacey, R., Cable, N., Bartley, M., . . . Delpierre, C. (2013). Childhood adversity as a risk for cancer: Findings from the 1958 British birth cohort study. BMC Public Health, 13(1), 767.

Kenne Sarenmalm, E., Browall, M., Persson, L. O., FallDickson, J., \& Gaston-Johansson, F. (2013). Relationship of sense of coherence to stressful events, coping strategies, health status, and quality of life in women with breast cancer. Psycho-Oncology, 22(1), 20-27.

Kiecolt-Glaser, J. K., \& Glaser, R. (1999). Psychoneuroimmunology and cancer: Fact or fiction? European Journal of Cancer, 35(11), 1603-1607.

Kronfol, Z., \& Remick, D. G. (2014). Cytokines and the brain: Implications for clinical psychiatry. American Journal of Psychiatry, 157(5), 683-694.

Kübler-Ross, E. (1969). On death and dying. New York, NY: Scribner.

Lambruschi, F., Lenzi, S., \& Leoni, F. (2004). Sviluppo del modello clinico cognitivista in età evolutiva. In F. Lambruschi (Ed.), Psicoterapia cognitiva dell'età evolutiva. Procedure di assessment e strategie psicoterapeutiche. Turin, Italy: Bollati Boringhieri.

Larkin, H., Felitti, V. J., \& Anda, R. F. (2014). Social work and adverse childhood experiences research: Implications for practice and health policy. Social Work in Public Health, 29, 1-16.

Laudenslager, M. L., Aasal, R., Adler, L., Berger, C. L., Montgomery, P. T., Sandberg, E., . . Reite, M. L. (1998). Elevated cytotoxicity in combat veterans with longterm post-traumatic stress disorder: Preliminary observations. Brain, Behavior, and Immunity, 12(1), 74-79.

Leonard, B. E., \& Song, C. (1996). Stress and the immune system in the etiology of anxiety and depression. Pharmacology, Biochemistry, and Behavior, 54(1), 299-303.

Liotti, G., \& Farina, B. (2011). Sviluppi traumatici. Eziopatogenesi, clinica e terapia della dimensione dissociativa. Milan, Italy: Cortina Raffaello.

Liotti G., \& Farina, B. (2012). Prefazione all'edizione italiana. In R. A. Lanius, E. Vermetten, \& C. Pain (Eds.), L'impatto del trauma infantile sulla salute e sulla malattia. L'epidemia nascosta [Trauma and illness: EMDR in psycho-oncology]. Rome, Italy: Giovanni Fioriti Editore.

Lyons-Ruth, K., Bronfman, E., \& Parsons, E. (1999). Atypical attachment in infancy and early childhood among children risk. IV. Maternal frightened, frightening, or atypical behavior and disorganized infant attachment patterns. Monographs of the Society for Research in Child Development, 64(3), 67-96.

Mager, W., \& Andrykowski, M. (2002). Communication in the cancer "bad news" consultation: Patient perceptions and psychological adjustment. Psycho-Oncology, 11, $35-46$.

Majidzadeh, A., \& Sediq, S. H. (2015a). Study descriptive static of desensitization technic efficiency with eye movement and reprocessed of cancer patient depression stress. International Science and Investigation Journal, 4(6), $11-17$.
Majidzadeh, A., \& Sediq, S. H. (2015 b). Study effective factors dedication static in cancer on cancer patient anxiety stress. International Science and Investigation Journal, 4(6), $1-10$.

Marshall, R. D., Olfson, M., Hellman, F., Blanco, C., Guardino, M., \& Struening E. L. (2001). Comorbidity, impairment, and suicidality in subthreshold PTSD. American Journal of Psychiatry, 158(9), 1467-1473.

Matsuoka, Y., Nagamine, M., \& Uchitomi, Y. (2006). Intrusion in women with breast cancer. In N. Kato, M. Kawata, \& R. K. Pitman (Eds.), PTSD: Brain mechanism and clinical implications (pp. 169-178). Tokyo, Japan: Springer-Verlag.

Matsuoka, Y., Yamawaki, S., Inagaki, M., Akechi, T., \& Uchitomi, Y. (2003). A volumetric study of amygdala in cancer survivors with intrusive recollections. Biological Psychiatry, 54(7), 736-743.

Matz, K., Junghöfer, M., Elbert, T., Weber, K., Wienbruch, C., \& Rockstroh, B. (2010). Adverse experiences in childhood influence brain responses to emotional stimuli in adult psychiatric patients. International Journal of Psychophysiology, 75, 277-286.

Maunsell, E., Brisson, C., Dubois, L., Lauzier, S., \& Fraser, A. (1999). Work problems after breast cancer: An exploratory qualitative study. Psycho-Oncology, 8(6), 467-473.

Mazzola, A., Calcagno, M. L., Goicochea, M. T., Pueyrredon, H., Leston, J., \& Salvat, F. (2009). EMDR in the treatment of chronic pain. Journal of EMDR Practice and Research, 3, 66-79.

Meaney, M. J. (2010). Epigenetics and the biological definition of gene $\mathrm{x}$ environment interactions. Child Development, 81(1), 41-79.

Meaney, M. J., \& Szyf, M. (2005). Environmental programming of stress responses through DNA methylation: Life at the interface between a dynamic environment and a fixed genome. Dialogues in Clinical Neuroscience, $7(2), 103-123$.

Mehnert, A., Berg, P., Henrich, G., \& Herschbach, P. (2009). Fear of cancer progression and cancer-related intrusive cognitions in breast cancer survivors. Psycho-Oncology, 18(12), 1273-1280.

Mehnert, A., \& Koch, U. (2007). Prevalence of acute and post-traumatic stress disorder and comorbid mental disorders in breast cancer patients during primary cancer care: A prospective study. Psycho-Oncology, 16(3), 181-188.

Monahan, K., \& Forgash, C. (2012). Childhood sexual abuse and adult physical and dental health outcomes. INTECH Open Access Publisher, 137-151.

Morasso, G., Di Leo, S., \& Grassi, L. (2002). La Psiconcologia: Stato dell'arte. In D. Amadori, M. L. Bellani, P. Bruzzi, P. G. Casali, L. Grassi, G. Morasso, \& W. Orrù (Eds.), Psiconcologia. Milan, Italy: Masson.

Morasso, G., \& Tomamichel M. (2005). La sofferenza psichica in oncologia. Modalità di intervento. Rome, Italy: Carocci Faber.

Nakano, T., Wenner, M., Inagaki, M., Kugaya, A., Akechi, T., Matsuoka, Y., . . U Uchitomi, Y. (2002). Relationship between distressing cancer-related recollections 
and hippocampal volume in cancer survivors. American Journal of Psychiatry, 159(12), 2087-2093. National Comprehensive Cancer Network. (2013). NCCN guidelines. Retrieved from https://www.nccn.org/professionals/ physician_gls/f_guidelines.asp

Nunes, S. O., Reiche, E. M., Morimoto, H. K., Matsuo, T., Itano, E. N., Xavier, E. C., . . Kaminami, M. S. (2002). Immune and hormonal activity in adults suffering from depression. Brazilian Journal of Medical and Biological Research, 35(5), 581-587.

Oberlander, T. F., Weinberg, J., Papsdorf, M., Grunau, R., Misri, S., \& Devlin, A. M. (2008). Prenatal exposure to maternal depression, neonatal methylation of human glucocorticoid receptor gene (NR3C1) and infant cortisol stress responses. Epigenetics, 3(2), 97-106.

O’Connor, A. P., Wicker, C. A., \& Germino, B. B. (1990). Understanding the cancer patient's search for meaning. Cancer Nursing, 13(3), 167-175.

Peters, E., Wissing, M. P., \& Du Plessis, W. F. (2002). Implementation of EMDR (R) with cancer patients: Research. Health SA Gesondheid, 7(2), 100-109.

Phipps, S., Klosky, J., Long, A., Hudson, M., Huang, Q., Zhang, H., \& Noll, R. (2014). Posttraumatic stress and psychological growth in children with cancer: Has the traumatic impact of cancer been overestimated? Journal of Clinical Oncology, 32(7), 641-646.

Posluszny, D., Edwards, R., Dew, M., \& Baum, A. (2011). Perceived threat and PTSD symptoms in women undergoing surgery for gynecologic cancer or benign conditions. Psycho-Oncology, 20(7), 783-787.

Rees, C. (2007). Childhood attachment. British Journal of General Practice, 57, 920-922.

Reiche, E. M., Nunes, S. O., \& Morimoto, H. K. (2004). Stress, depression, the immune system, and cancer. Lancet Oncology, 5(10), 617-625.

Roberts, B. W., Wood, D., \& Smith, J. L. (2005). Evaluating five factor theory and social investment perspectives on personality trait development. Journal of Research in Personality, 39, 166-184.

Rodenburg, R., Benjamin, A., Meijer, A. M., \& Jongeneel, R. (2009). Eye movement desensitization and reprocessing in an adolescent with epilepsy and mild intellectual disability. Epilepsy \& Behavior, 16(1), 175-180.

Rodin, G., Lo, C., Mikulincer, M., Donner, A., Gagliese, L., \& Zimmermann, C. (2009). Pathways to distress: The multiple determinants of depression, hopelessness, and the desire for hastened death in metastatic cancer patients. Social Science \& Medicine, 68(3), 562-569.

Rodin, G., Zimmermann, C., Rydall, A., Jones, J., Shepherd, F. A., Moore, M., . . G Gagliese, L. (2007). The desire for hastened death in patients with metastatic cancer. Journal of Pain and Symptom Management, 33, 661-675.

Roth, T. L., \& Sweatt, J. D. (2011). Epigenetic marking of the BDNF gene by early-life adverse experiences. Hormones and Behavior, 59(3), 315-320.

Rowland, J. H., Desmond, K. A., Meyerowitz, B. E., Belin, T. R., Wyatt, G. E., \& Ganz, P. A. (2000). Role of breast reconstructive surgery in physical and emotional outcomes among breast cancer survivors. Journal of the National Cancer Institute, 92(17), 1422-1429.

Saccomani, R. (1998). Tutti bravi. Psicologia e clinica del bambino portatore di tumore. Milan, Italy: Cortina Rafaello.

Sapolsky, R. M., \& Meaney, M. J. (1986). Maturation of the adrenocortical stress response: Neuroendocrine control mechanisms and the stress hyporesponsive period. Brain Research, 396(1), 64-76.

Schmale, A. H., Jr., \& Engel, G. L. (1967). The giving upgiven up complex illustrated on film. Archives of General Psychiatry, 17, 135-145.

Schneider, J., Hofmann, A., Rost, C., \& Shapiro, F. (2007). EMDR in the treatment of chronic phantom limb pain. Pain Medicine, 9(1), 76-82.

Schore, A. N. (2000). Attachment and the regulation of the right brain. Attachment \& Human Development, 2(1), 23-47.

Schore, A. N. (2003). Affect dysregulation and the disorders of the self/affect regulation and the repair of the self. New York, NY: Norton.

Schore, A. N. (2009). Relation trauma and the developing right brain: An interface of psychoanalytic self psychology and neuroscience. Annals of the New York Academy of Sciences, 1159, 189-203.

Shapiro, F. (1995). Eye movement desensitization and reprocessing: Basic principles, protocols, and procedures. New York, NY: Guilford Press.

Shapiro, F. (2001). The challenges of treatment evolution and integration. American Journal of Clinical Hypnosis, 43(3-4), 183-186.

Shapiro, F. (2014). The role of eye movement desensitization and reprocessing (EMDR) therapy in medicine: Addressing the psychological and physical symptoms stemming from adverse life experiences. The Permanente Journal, 18, 71-77.

Shelby, R. A., Golden-Kreutz, D. M., \& Andersen, B. L (2008). PTSD diagnoses, subsyndromal symptoms, and comorbidities contribute to impairments for breast cancer survivors. Journal of Traumatic Stress, 21(2), 165-172.

Shin, L. M., Rauch, S. L., \& Pitman, R. K. (2006). Amygdala, medial prefrontal cortex, and hippocampal function in PTSD. Annals of the New York Academy of Sciences, 1071, 67-79.

Shirtcliff, E. A., Coe, C. L., \& Pollak, S. D. (2009). Early childhood stress is associated with elevated antibody levels to herpes simplex virus type 1. Proceedings of the $\mathrm{Na}$ tional Academy of Sciences of the United States of America, 106(8), 2963-2967.

Shonkoff, J. P., Garner, A. S., Siegel, B. S., Dobbins, M. I., Earls, M. F., McGuinn, L., . . W Wood, D. L. (2012). The lifelong effects of early childhood adversity and toxic stress. Pediatrics, 129(1), e232-e246.

Smith, M. Y., Redd, W. H., Peyser, C., \& Vogl, D. (1999). Post-traumatic stress disorder in cancer: A review. Psycho-Oncology, 8(6), 521-537.

Smith, S. K., Zimmerman, S., Williams, C. S., Benecha, H., Abernethy, A. P., Mayer, D. K., . . Ganz, P. A. (2011). 
Post-traumatic stress symptoms in long-term nonHodgkin's lymphoma survivors: Does time heal? Journal of Clinical Oncology, 29(34), 4526-4533.

Söllner, W., DeVries, A., Steixner, E., Lukas, P., Sprinzl, G., Rumpold, G., \& Maislinger, S. (2001). How successful are oncologists in identifying patient distress, perceived social support, and need for psychosocial counselling? British Journal of Cancer, 84, 179-185.

Stein, M. B., Walker, J. R., Hazen, A. L., \& Forde, D. R. (1997). Full and partial posttraumatic stress disorder: Findings from a community survey. American Journal of Psychiatry, 154, 1114-1119.

Tesarz, J., Leisner, S., Gerhardt, A., Janke, S., Seidler, G. H., Eich, W., Hartmann, M. (2014). Effects of eye movement desensitization and reprocessing (EMDR) treatment in chronic pain patients: A systematic review. Pain Medicine, 15, 247-263.

Tessier, P., Lelorain, S., \& Bonnaud-Antignac, A. (2012). A comparison of the clinical determinants of healthrelated quality of life and subjective well-being in longterm breast cancer survivors. European Journal of Cancer Care, 21(5), 692-700.

Thompson, S., Eccleston, L., \& Hickish, T. (2011). Posttraumatic stress disorder in cancer survivors: Recognising and acknowledging the symptoms. WebmedCentral Oncology 2011, 2(8), 1-16.

Townsend, A., Wyke, S., \& Hunt, K. (2006). Self-managing and managing self: Practical and moral dilemmas in accounts of living with chronic illness. Chronic Illness, 2(3), 185-194.

Van der Kolk, B. A. (2003). Posttraumatic stress disorder and the nature of trauma. In M. F. Solomon \& D. J. Siegel (Eds.), Healing trauma: Attachment, mind, body, and brain (pp. 168-195). New York, NY: Norton.
Van der Kolk, B. A., McFarlane, A. C., \& Weisaeth, L. (2005). Stress traumatico. Gli effetti sulla mente, sul corpo e sulla società delle esperienze intollerabili [Traumatic stress: The effects of overwhelming experience on mind, body, and society]. Rome, Italy: Magi Edizioni.

Varetto, A., Ramonda, E., Stanizzo, M. R., \& Torta, R. (2007). L'approccio psicoterapeutico alla malattia oncologica. In R. Torta \& A. Mussa (Eds.), PsicOncologia. Il modello biopsicosociale (2nd ed.). Turin, Italy: Centro Scientifico Editore.

Watson, M., Haviland, J. S., Greer, S., Davidson, J., \& Bliss, J. M. (1999). Influence of psychological response on survival in breast cancer: A population-based cohort study. Lancet, 354, 1331-1336.

Watson, M., Homewood, J., Haviland, J., \& Bliss, J. M. (2005). Influence of psychological response on breast cancer survival: 10-year follow-up of a population-based cohort. European Journal of Cancer, 41, 1710-1714.

Wegman, H. L., \& Stetler, C. (2009). A meta-analytic review of the effects of childhood abuse on medical outcomes in adulthood. Psychosomatic Medicine, 71(8), 805-812.

Whitaker, K. L., Brewin, C. R., \& Watson, M. (2008). Intrusive cognitions and anxiety in cancer patients. Journal of Psychosomatic Research, 64(5), 509-517.

Yoshikawa, E., Matsuoka, Y., Yamasue, H., Inagaki, M., Nakano, T., Akechi, T., . . Uchitomi, Y. (2006). Prefrontal cortex and amygdala volume in first minor or major depressive episode after cancer diagnosis. Biological Psychiatry, 59(8), 707-712.

Zani, B., \& Cicognani, E. (1999). Le vie del benessere. Eventi di vita e strategie di coping. Rome, Italy: Carocci Editore.

Merci d'adresser toute correspondance concernant cet article à Elisa Faretta, PIIEC Centre Study, Via Settembrini 56, 20124, Milan, Italie. Courriel : e.faretta@piiec.com 